\title{
Carnets
}

Revue électronique d'études françaises de l'APEF

Deuxième série - 21 | 2021

Arts du vin

\section{In nomine vini, et corporis, et spiritus sancti}

Ainsi soit-il dans l'œuvre de François Rabelais

\section{Frédéric-Gaël Theuriau}

\section{(2) OpenEdition}

Journals

Édition électronique

URL : https://journals.openedition.org/carnets/12940

DOI : $10.4000 /$ carnets. 12940

ISSN : 1646-7698

Éditeur

APEF

Référence électronique

Frédéric-Gaël Theuriau, "In nomine vini, et corporis, et spiritus sancti », Carnets [En ligne], Deuxième série - 21 | 2021, mis en ligne le 31 mai 2021, consulté le 01 juin 2021. URL : http://

journals.openedition.org/carnets/12940; DOI : https://doi.org/10.4000/carnets.12940

Ce document a été généré automatiquement le 1 juin 2021.

\section{(c) (7) 8}

Carnets est mis à disposition selon les termes de la licence Creative Commons - Atribution - Pas d'utilisation commerciale 4.0 International. 


\title{
In nomine vini, et corporis, et spiritus sancti
}

\author{
Ainsi soit-il dans l'œuvre de François Rabelais
}

Frédéric-Gaël Theuriau

\section{NOTE DE L'AUTEUR}

Cet article est le fruit d'une recherche concernant les humanités médicales ainsi que les rapports entre littérature et médecine effectuée dans le cadre du projet $n^{\circ} 2018045$ intitulé « Médecine narrative dans l'humanisme médical : dialectique du médecin, de la maladie et du patient » ayant eu l'avis favorable de la Commission Éthique en Recherche Humaine du CHRU de Tours et dont le responsable de la recherche est l'auteur du présent article. La Radio Chrétienne francophone, dans le cadre de son émission hebdomadaire « La saga des siècles » du 25 novembre 2009, évoqua le thème « Rabelais et le vin » issu de ce travail de recherche.

\section{La soif de savoir}

1 L'Humanisme peut se définir comme un courant interstitiel où transitent à la fois le matériau médiéval obscur et les futures évolutions modernes, et qui présente en outre les caractéristiques d'une révolution idéologique au sujet d'une nouvelle représentation érudite et éthique du monde, de la société et de l'Homme. En matière d'éducation, il intègre la double formation de l'esprit et du corps qu'il valorise dans cette devise tirée des derniers vers de la satire $\mathrm{x}$ des Saturae de Juvénal : Mens sana in corpore sano.

2 L'éducation du prince proposée par François Rabelais dans son Gargantua ${ }^{1}$ répond à cette maxime de "l'esprit sain dans un corps sain ", idéal de sagesse expliqué par le poète satirique latin. Plus précisément, l'œuvre du moine-médecin-écrivain présente une réflexion indirecte autour de la dimension physique de l'Être, le corps qui, en 
dehors des besoins liés à la respiration et à l'élimination des déchets, doit régulièrement se nourrir, donc manger et boire pour vivre.

S'il est connu que l'absorption de boisson symbolise le savoir érudit et curieux, le but est de définir les effets du vin sur le corps et l'esprit dans l'œuvre de Rabelais un tantinet épicurien au milieu des vignobles chinonais².

4 Les questions vitale, religieuse et médicale définiront ainsi les enjeux du vin dans son œuvre centrée sur le Traité de bon usage de vin ${ }^{3}$ et en lien avec Pantagruel, Gargantua, Le Tiers Livre, Le Quart Livre et Le Cinquiesme Livre.

\section{La question vitale}

Depuis les débuts de l'histoire humaine, avec l'arrivée de l'écriture hiéroglyphique, la bière était la boisson quotidienne bon marché, fabriquée à partir de la fermentation de blé, d'orge, de pain, d'épices et de dattes, aussi bien pour les pauvres que les riches. Le vin, plus complexe à fabriquer, fit ensuite son apparition dont l'origine serait le Caucase vers moins 6000 .

6 Les échanges commerciaux avec les civilisations bénéficiant d'un climat méditerranéen et propice permirent l'introduction de la vigne, en Égypte, qui réussit en partie à s'adapter au nouveau sol. La viticulture demandait cependant un savoir faire et une attention plus soutenus avant l'étape de la viniculture. La rareté du vin, qui engendra un art de boire ritualisé et mesuré, en faisait un marqueur social réservé à l'aristocratie princière comme en témoignent les représentations de jarres à vin et de la culture de la vigne retrouvées datant de la fin du IV millénaire avant notre ère.

7 D'après Platon, son usage était soumis à des règles variables en fonction des moments de la vie. Les jeunes de moins de dix-huit ans ne devaient pas le boire. Ensuite, jusqu'à quarante ans environ, le vin ne devait pas être absorbé avec excès. L'ivresse démesurée était finalement réservée à la vieillesse. Dans le Timée (58-60), le philosophe écrivit, au sens propre, que ce «qui réchauffe l'âme en même temps que le corps est le vin "4. Peu après, Aristote, dans Les Problèmes, expliquait que le vin causait les mêmes effets que la bile noire, à l'origine de la mélancolie, dont sont atteints certains esprits géniaux, maladie atteignant l'une des quatre humeurs, générant des effets plus ou moins désirables. Aristote qui n'en condamne pas l'usage montre, dans Les Problèmes (xxx, III), que le vin agit sur l'ensemble de l'être.

8 François Rabelais avait parfaitement connaissance de ces représentations ancestrales, érudit qu'il était dans tous les domaines, imprégné de littérature antique et persuadé qu'un retour à certaines valeurs ancestrales pouvait élever l'individu et favoriser la construction d'une société plus harmonieuse. Ne rappelle-t-il d'ailleurs pas, dans son Traité de bon usage de vin, une remarque de Platon? En effet, le sous-titre exprime directement la pensée platonicienne sur le vin capable d'« ébaudir âme et corps ", c'est-àdire d'égayer âme et corps. En conséquence, d'après le sous-titre, en ce qui concerne le corps, le vin aurait la vertu de le mettre dans un état de mouvement traduisant la liesse. Il vante le vin car il ne condamne pas le corps pour exalter l'esprit.

9 Pour ce qui concerne les "membres extérieurs \& intérieurs », dans la suite du sous-titre, qui rappellent la notion de corps remise à l'honneur par Rabelais en opposition directe à l'esprit scolastique, Galien, au $\mathrm{II}^{\mathrm{e}}$ siècle, définissait trois "choses naturelles »: les membres, les esprits physiques, le sang. Une action ciblée sur ces « choses » permettait 
d'atteindre un état d'équilibre comme une balance. Pour cela, divers éléments étaient considérés comme agissants : l'air, la nourriture, la boisson, le mouvement, le repos, le sommeil, la veille, l'inanition, la réplétion, les passions. La boisson est l'élément retenu par Rabelais dans le Traité. Son titre, riche d'informations, en suggère un usage particulier : il s'agit du «bon usage ». De ce fait, il ne s'agit nullement d'inciter les gens à boire avec surabondance mais d'apprendre à boire bien en société et non pas mal afin que s'instaure une vitalité équilibrante et socialisante du corps. L'usage de l'eau est prohibé par Rabelais qui préfère le vin pur, source d'un corps bien sculpté à la manière d'un bodybuilder: " une verge puissante et belle ", "le ventre et le bras musculeux », «[des] jambes (...) telles des mâts de vaisseaux» (Rabelais, 2016: 40-41), autant de qualités physiques qui témoignent d'une vitalité extraordinaire.

Rabelais doit être envisagé dans son contexte pour évaluer son rapport au vin et ses effets sur le corps. Il faut savoir que la culture de l'ivresse et de l'enivrement remonte au Moyen Âge en France comme le montrent les chansons bachiques d'olivier Basselin cité par Rabelais en conclusion de son Traité. Les années 1530 marquèrent le début d'un renversement progressif des pratiques et des valeurs, et Rabelais se situe à l'interstice de deux conceptions antagonistes ${ }^{5}$. Les autorités comme l'État, l'Église et les médecins ${ }^{6}$, dont Rabelais peut-être, condamnèrent l'ivrognerie publique ${ }^{7}$. François $\mathrm{I}^{\text {er }}$ promulgua un édit, daté du $1^{\mathrm{er}}$ août 1536, où étaient signifiées les peines encourues : amputation des oreilles, infamie, bannissement. Dans son Traité, Rabelais s'amuse à défier l'autorité en disant: "Que nous chaut (...) une oreille» (Rabelais, 2016: 23). Autrement dit, l'ivrognerie publique peut aboutir à l'amputation d'une partie du corps et donc à une diminution de la vitalité. En pratique, la loi ne fut pas appliquée ${ }^{8}$ mais une lutte indirecte s'instaura en cas de troubles à l'ordre public au niveau des débits de boissons dont Rabelais établit une liste dans son Traité : « cabarets, auberges, tavernes, gargotes, estaminets et cambuses » (Rabelais, $2016: 22$ ).

11 Ainsi, depuis la nuit des temps, le vin apparaît comme un symbole de réussite sociale à cause de la noblesse de ses origines. Rabelais en fait un marqueur culturel populaire fort lié à une mobilité particulière du corps en s'inscrivant dans l'idée que le vin est vital $^{9}$, et il le fait descendre des strates sociales élevées vers les plus humbles. Placé sous l'auspice de « Maître Alcofribas, l'architriclin du grand Pantagruel » qui clôt le sous-titre, le vin est semble-t-il également lié à la religion.

\section{La question religieuse}

12 Dans la Bible, l'Évangile selon Saint Jean (II, 1-11) rapporte, qu'au cours des noces de Cana, le vin manquait. Jésus demanda à des serviteurs de remplir d'eau six jarres vides. Au moment de la goûter, l'architriclin, c'est-à-dire l'organisateur du repas, s'aperçut que c'était du vin qu'il buvait, symboliquement le sang du Christ. Ce fut là le premier miracle étonnant de Jésus en Galilée qui montre ainsi sa gloire et ses disciples croient en lui.

Le cours de la vie d'un croyant est rythmé par divers sacrements qui doivent trouver grâce devant Dieu ${ }^{10}$. Au chapitre XxxI du Tiers livre, une discussion s'instaure entre Panurge et le médecin Rondibilis. Panurge l'interroge sur la fidélité dans le mariage. Le sujet, qui pourrait déboucher sur une réponse sérieuse autour d'un engagement et d'une promesse maintenue par l'Esprit-Saint ou encore autour d'un conseil en lien avec 
un précepte de l'amour courtois établi dans le De Arte honeste amandi d'Andreas Capellanus (1186), est d'une toute autre nature. Il est pour ainsi dire tourné en dérision.

L'interrogation déclenche en effet une plus ou moins claire et savante réponse ancrée sur une connaissance des thèses des Anciens comme Platon, Diodore de Sicile, Plutarque et des contemporains comme Nicolas de la Chesnaye et André Tiraqueau. Selon Rondibilis, l'attirance sexuelle est réfrénée par cinq facteurs. L'un d'entre eux est le vin : "par l'intemperance du vin, advint au corps humain refroidissement de sang, resolution des nerfs, dissipation de semence generative, hebetation des sens, perversion des mouvements : qui sont toutes impertinences à l'acte de generation " (Rabelais, 1961: 143). Il ajoute ensuite qu'«[a]ultrement est du vin prins temperement » (Rabelais, 1961 : 143). De ce fait, il y a donc deux manières de boire, la mauvaise qui mettrait en péril l'action reproductrice chez l'homme et la bonne qu'il recommande expressément afin que le corps puisse pleinement consommer l'amour physique.

15 L'abus du vin qui semble prohibé, c'est-à-dire la surabondance, est encore dénoncée dans Le Cinquiesme Livre, au chapitre xxxIv. Si le vin permet une interaction entre les choses terrestres et spirituelles, sa consommation permet d'ouvrir les esprits à une certaine spiritualité, voire à une vertu inspiratrice. Mais il faut qu'elle soit limitée car ceux « qui s'addonnent et dedient à contemplation des choses divines doivent en tranquillité leurs esprits maintenir, hors toute perturbation de sens : laquelle plus est manifestée en yvrognerie qu'en autre passion, quelle que soit » (Rabelais, 1948 : 137).

Lorsque Rabelais semble cheminer sur des chemins matérialistes, le spirituel n'est finalement jamais éloigné comme dans son allusion au début du Traité: «L'usage du vin, outre le verbe prolixe et la prière fervente, est de toutes les actions humaines " (Rabelais, 2016 : 21). Certes l'on s'éloigne du corps pour se rapprocher de l'âme ; mais la contiguïté avec la "corporation des buveurs pantagruélistes» (Rabelais, 2016:21) rappelle une autre dimension corporelle, celle des buveurs regroupés en un corps associatif et ayant besoin d'une ligne directrice tournée sur le "salut ${ }^{11}$ divin puisque le premier chapitre est titré «Paix en Jésus le Christ notre Sauveur » (Rabelais, 2016 : 21).

Rabelais engage généralement à boire modérément sous la protection de Bacchus, comme il l'explique au chapitre LXv du Quart Livre, pour « hault eslevez les esprits des humains, leurs corps evidentement alaigriz, et assouply ce que en eulx estoit terrestre » (Rabelais, 1959: 237), traduisant, par cela même, la recherche du mystère de l'Eucharistie. En effet, le vin est toujours envisagé dans le sens propre comme dans le figuré. Pantagruel, qui navigue à la recherche du Temple de la Dive bouteille, tel un Ulysse ou un chevalier en quête du Saint Graal, prétend que le corps privé de nourriture et de boisson est terrestre tandis que celui qui est rassasié est plus enclin à s'élever. Non seulement le vin est l'allégorie du savoir livresque, mais encore est-il celui $\mathrm{du}$ sang du Christ dans la liturgie eucharistique où il est question d'élever son cœur en absorbant le corps et le sang du Christ, c'est-à-dire le pain sans levain et le vin, est-il mentionné dans l'Évangile selon Saint Marc (xIV, 12-26).

Cela se confirme dans Le Cinquiesme Livre qui concerne la finalité des aventures de Pantagruel. Au livre xuv, l'oracle de la Dive bouteille, la prêtresse Bacbuc, dans son interprétation du mot de la Dive bouteille, affirme à Panurge que " non rire, ains boire est le propre de l'homme » (Rabelais, 1948: 169), ce qui va à l'encontre de la remarque d'Aristote traduit au dernier vers du dizain en tête du Gargantua qui est « que rire est le 
propre de l'homme » (Rabelais, $1996: 45)$. La contradiction n'est qu'apparente si l'on considère le bon vin stimulant le corps en proie à un certain engouement, $\mathrm{y}$ compris langagier, conduisant à une métaphore de la science où l'homme peut s'abreuver pour acquérir la connaissance suffisante pour décider par lui-même, devenir plus libre et être mieux responsable de ses actes, autant de qualités humanistes recherchées.

Le vin, sorte de quintessence intellectuelle, spirituelle ou mystique, n'est pas seulement envisagé comme allusions bibliques pour jouer avec les mots afin de cacher un sens plus élevé et sérieux au sujet de l'élévation corporelle vers le royaume des Cieux. Rabelais est aussi conscient que l'œnothérapie a peut-être sa place puisque la suite du sous-titre du Traité concerne l'effet protecteur «contre diverses maladies de membres extérieurs \& intérieurs ».

\section{La question médicale}

Le vin pourrait ainsi guérir deux parties du corps humain, c'est-à-dire les membres et les organes. Le constat des qualités thérapeutiques du vin serait d'ailleurs apparu dès le $\mathrm{IV}^{\mathrm{e}}$ millénaire avant notre ère. À partir $\mathrm{du} \mathrm{IV}^{\mathrm{e}}$ siècle avant notre ère, le corpus hippocraticum exposait les bienfaits du vin comme remèdes à divers maux. Or, Rabelais avait une bonne connaissance des thèses hippocratiques et galiennes par sa connaissance du grec ancien, sans parler, par celle de l'arabe, de la médecine avicennienne et averroïste, où la corporéité est envisagée ${ }^{12}$. On le voit bien dans le chapitre v du Gargantua avec «Les propos des bienyvres » qui emplissent leur estomac de bon vin pineau.

21 Pour le médecin Rabelais, c'est en réalité l'ignorance qui est la fautive sur l'usage du vin, d'où la visée du Traité d'instruire sur les règles de son emploi. L'auteur, qui avait obtenu son doctorat en médecine en 1537, lorsqu'il parle du vin et de ses excès, doit finalement être compris comme une manière ironique de dénoncer les méfaits du vin pris sans réflexion avec ses effets néfastes sur le corps parfois déformé et détruit. L'ivrognerie conduit, malgré l'émergence de paroles savantes, à la déconstruction logique du dialogue comme le démontre le chapitre v du Gargantua.

Quant au Traité, au chapitre III, il n'évoque que les aspects bénéfiques et ne présente que les indications thérapeutiques bonnes pour soigner certaines parties du corps, non sans jeux de mots, doubles sens et triples sens laissés au détour d'une expression. Il est donc évident que les appellations des maladies renvoient à la fois à de véritables maux mais aussi à des situations cocasses qui mesurent et nuancent les propos soi-disant sérieux du narrateur du traité, "Maître Alcofribas, l'architriclin du grand Pantagruel ». La bonne action supposée du vin sur une maladie est toujours liée à une partie anatomique du corps. Un parallèle entre la vision de la maladie au $\mathrm{XVI}^{\mathrm{e}}$ siècle et sa représentation actuelle est possible à établir malgré des terminologies médicales différentes à l'époque.

23 Les bienfaits du vin sur la santé physique est actuellement une étude très sérieuse. Le champagne par exemple, avec la présence de sels minéraux et d'oligo-éléments joue un rôle antidépresseur et anxiolytique. Le vin s'attaque au mauvais cholestérol souvent responsable de maladies coronariennes. Le vin rouge pourrait aussi lutter efficacement contre certains cancers - poumon, prostate, ovaires - grâce à la présence de substances comme les polyphénols et éviter la dégénérescence neuronale. Sans parler des vertus 
antibactériennes connues depuis le milieu du XIX ${ }^{e}$ siècle. Il existe un tableau mettant en évidence les qualités thérapeutiques du vin sur les fonctions physiologiques: Acidose (Sancerre), Anémie (Pomerol), Constipation (Anjou, Vouvray), Diarrhée (Madiran), Digestion (Champagne), Gastralgie (Sauternes), Uricémie (Sancerre), etc. Le Traité de bon usage de vin ressemble, par certains égards, à cette analyse des bienfaits du vin pris modérément sur certaines parties du corps, constat d'ailleurs fait par Pasteur au XIX siècle et par la médecine actuelle. Dans le traité, Rabelais l'évoque aussi mais l'on ne sait si c'est sérieux ou non: "Buvez donc du vin (...) par-dessus tout contre le dessèchement du cerveau dans le crâne et contre la jaunisse. Contre divers maux, préférez les vins souscrits : de Gascogne contre la gale, de Bourgogne contre la rogne » (Rabelais, 2016:27,29). Sans parler des actions bénéfiques du vin ajouté à des herbes ou à des plantes comme " la cannelle ", « la sauge » et " autres ingrédients " (Rabelais, 2016 : 29).

Il apparait donc que François Rabelais utilise ses connaissances médicales pour établir ses parallèles entre le corps et le vin d'un point de vue médical, sans doute le fruit de ses observations et de ses connaissances, ce qui place le vin en tant que remède malgré un arrière plan qui laisse imaginer une farce sur tout ce qu'il vient de mentionner.

\section{Stellogenèse créatrice du vin, du corps et du spirituel}

Dans le Traité de bon usage de vin, lequel est grand \& perpétuel pour ébaudir âme \& corps \& contre diverses maladies de membres extérieurs \& intérieurs composé au profit d'enlumineurs de museaux par maitre Alcofribas, l'architriclin du grand Pantagruel, le narrateur Alcofribas annonce son projet de consigner, suivant les préceptes de Pantagruel, ce qu'il convient de retenir sur le vin. Trois axes apparaissent d'emblée dans le titre long, à l'instar du reste de son œuvre : les dimensions vitale, religieuse et médicale ${ }^{13}$.

Les effets du vin sur le corps, dans le Traité et son œuvre en général, est peu flatteur pour le corps humain même si le mode est celui du comique. Pourtant il célèbre le vin dès les premières lignes du Pantagruel, en 1532. Mais ce fut l'année où l'été fut si terrible que la sécheresse eut pour conséquence une pénurie d'eau. Pour éviter la déshydratation, seule solution: boire du vin. Cette solution ne devait d'ailleurs sans doute servir à rien car le vin ne permet pas de s'hydrater. Mais pour lui, le vin fait partie du bon vivre en société, le vin participe au mystère de la Foi, le vin est une médication contre certains maux, tels sont les trois effets principaux du vin pris à bon escient. Loin d'encourager l'ivrognerie, Rabelais chemine sur un art de boire bénéfique pour le corps et l'esprit et préfère rire que pleurer des usages excessifs. Le vin qui passe d'abord dans le corps aboutit nécessairement au spirituel puisque le terme "spiritueux », qui désigne une boisson alcoolisé obtenue par distillation, vient du latin spiritus, -us, m signifiant "esprit». Le corps agit donc chez Rabelais comme un distillateur alchimique d'une boisson dont le produit permet de rapprocher l'âme de Dieu.

27 C'est ainsi que s'établit une sorte d'équilibre entre mises en garde et encouragements dissimulés derrière un faisceau infini de significations rayonnantes. Les mots, les néologismes, les expressions renvoient souvent à des significations multiples à la fois, dans toutes les directions et à des niveaux divers et variés, à la manière des rayons lumineux d'une étoile. Ils participent à l'essence même de la création, au processus de formation primordial du texte rabelaisien. Ce procédé de "stellogenèse créatrice $»^{14}$ 
présent chez Rabelais crée un tissu d'interprétations multiples. Quoi qu'il en soit il faut aussi rapprocher la métaphore du vin à celle du pantagruélion du Tiers Livre, le chanvre - plante qui a donné le cannabis médical -, dont les effets s'apparentent à une sorte d'ivresse.

\section{BIBLIOGRAPHIE}

BOQUET, Damien et NAGY Piroska (2015). Sensible Moyen Âge : Une histoire des émotions dans l'Occident médiéval. Paris : Seuil.

BOYANCÉ, Pierre (décembre 1951). «Platon et le vin », Bulletin de l'Association Guillaume Budé, nº 10, pp. 3-19.

ÉRASME (2016). Éloge de la folie. Paris : Flammarion.

LECOUTRE, Mathieu (2011). Ivresse et ivrognerie dans la France moderne. Renne/Tours : Presses Universitaires de Rennes/Presses Universitaires François-Rabelais.

LEFRANC, Abel (1924). « Le vin chez Rabelais », Revue du seizième siècle, t. 11, pp. 59-78.

PLATON (1925). CEuvres complètes, Timée. Paris : Les Belles Lettres.

RABELAIS, François (1948). CEuvres complètes, Le Cinquiesme Livre. Paris : Les Belles Lettres.

RABELAIS, François (1959). CEuvres complètes, Le Quart Livre. Paris : Les Belles Lettres.

RABELAIS, François (1961). CEuvres complètes, Le Tiers Livre. Paris : Les Belles Lettres.

RABELAIS, François (1996). Gargantua. Paris : Seuil.

RABELAIS, François (2012). Pantagruel. Paris : Hatier.

RABELAIS, François (2016). Traité de bon usage de vin, lequel est grand \& perpétuel pour ébaudir âme \& corps \& contre diverses maladies de membres extérieurs \& intérieurs composé au profit d'enlumineurs de museau par maître Alcofribas, l'architriclin du grand Pantagruel. Paris : Allia.

TALLET, Pierre (2008). « Une boisson destinée aux élites : le vin en Égypte ancienne », Revue de l'Académie des Inscriptions et Belles-Lettres, $\mathrm{n}^{\circ}$ 19, pp. 39-51.

THEURIAU, Frédéric-Gaël (2012). « Représentations littéraires de l'ivresse », in Daniel Leuwers (org.). L'Ivresse littéraires. Paris : Calliopées, pp. 39-42.

THEURIAU, Frédéric-Gaël (2017), «Stellogenèse créatrice dans le concept du narratoème », in Frédéric-Gaël Theuriau (éd.). Réfléchir sur l'œuvre de Hédi Bouraoui. Antibes : Vaillant, pp. 97-196.

\section{NOTES}

1. François Rabelais, Gargantua, chapitres XIV À XXIV, éd. 1542.

2. Rabelais vécut, durant son enfance, à La Devinière, à Seuilly, en Touraine, un logis bourgeois entouré de plusieurs hectares de vignobles répartis entre La Vienne et La Loire. Cette métairie 
était la maison de campagne de son père, Antoine Rabelais, avocat à Chinon. Elle se situait à l'origine au lieu-dit Les Cravandières dont le nom venait des bernaches cravants, des petites oies qui devaient passer par là pour rejoindre l'estuaire de la Loire au moment de la migration. Or, leur vol, dit-on, inspirait, dans leurs prédictions, les devins, d'où l'invention du nom « Devinière ».

3. Il faut cependant relativiser les termes et expressions du Traité de bon usage de vin dans la mesure où il s'agit d'une traduction en français du Xxi ${ }^{e}$ siècle (2009) exécutée à partir d'un texte tchèque (par Martin Carchesius, clerc du chancelier de Prague) de 1622 et non de l'époque de Rabelais.

4. Platon expose les différentes variétés des corps dont l'eau et ses « sucs » dérivés, en particulier

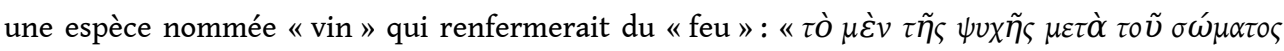

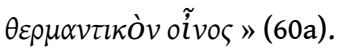

5. Rabelais est à rapprocher d'Érasme qui établissait, dans l'Éloge de la folie (1511), un rapport entre l'ivresse et la folie.

6. La dénonciation des méfaits de l'abus d'alcool, sachant que le taux d'alcool dans le vin à cette époque était très inférieur à aujourd'hui, va croissante jusqu'au $\mathrm{XVII}^{\mathrm{e}}$ siècle. Même si les vins étaient obtenus par le processus de fermentation du raisin (vinification), leur qualité était médiocre et ils étaient souvent servis avec de l'eau et des plantes ou des fruits macérés. Le terme «alcool », qui provient de l'arabe de l'époque médiévale, n'arrive en France et dans la conscience de sa présence dans le vin qu'au cours du $\mathrm{XvI}^{\mathrm{e}}$ siècle. Ainsi, peu de gens savant que ce n'est pas le vin proprement dit qui fait tourner les têtes mais l'éthanol (alcool éthylique) obtenu après transformation du jus de raisin sucré.

7. L'ivrognerie privée est difficile à mesurer bien qu'elle soit dénoncée sur le plan moral et social.

8. En raison des difficultés de la mise en place d'un système de répression directe.

9. Au contraire, la science actuelle a prouvé que la consommation de vin n'a absolument rien de vital chez l'Homme.

10. Il y a d'abord les sacrements d'initiation (baptême, confirmation et eucharistie), de guérison (réconciliation et onction) et du service (mariage ou ordination).

11. Le mot « salut » pourrait bien avoir deux sens : bonjour et salut divin.

12. Dans les années 1520, Rabelais s'était perfectionné dans le grec ancien et en arabe littéraire malgré les restrictions et les misères causées par la Sorbonne qui était alors une Faculté de théologie. Il fut cependant soutenu à partir de 1525 par son Évêque Geoffroy d'Estissac qui était son protecteur. Il put ainsi lire directement les écrits d'Hippocrate, de Galien, d'Avicenne et d'Averroès dans la langue originelle, éludant les fallacieuses traductions scolastiques. Candidat à la licence à la faculté de médecine de Montpellier en 1531, il fut chargé d'un enseignement et dispensa des cours basés sur les commentaires d'après les textes originaux des médecins grecs et arabes, sans passer par les mauvaises traductions, restituant donc mieux leurs pensées.

13. Dans le Prologue du Gargantua, Rabelais annonce son projet de dissimuler les codes symboliques de la lecture de ses œuvres de portée humaniste éminemment épico-satiricocomique. Son aphorisme natura abhorret vacuum essaime une problématique humaniste fondamentale qui caractérise l'Homme depuis les origines : ce dernier a toujours eu besoin de savoir pour comprendre le monde curieux qui l'entoure, il a toujours eu horreur de ne pas savoir et met tout en œuvre pour combler ses ignorances et résoudre ses interrogations comme le joyeux buveur qui étanche sa soif en buvant le vin vital à sa survie dans un monde qui le dépasse. 14. Le concept fut d'abord mis en évidence dans les narratoèmes d'Hédi Bouraoui (1932-) dans une tonalité transhumaniste, c'est-à-dire dans le sens d'un humanisme moderne qui traverse les différentes cultures pour promouvoir la paix, donc d'un humanisme qui traverse les cultures. 


\section{RÉSUMÉS}

Le vin est un enjeu littéraire omniprésent dans l'œuvre de François Rabelais. Le chantre de l'Humanisme le transcende pour servir de fondement à un idéal de vie en prétendant qu'il aurait la vertu « d'emplir l'ame de toute verité, tout savoir et philosophie » (Rabelais, Le Cinquiesme livre, chapitre XLV). Le méconnu Traité de bon usage de vin expose le projet d'un savoir-vivre dans lequel l'auteur explique la valeur et la place accordée au vin.

Wine is a literary issue which is represented in the literary work of François Rabelais. The defender of Humanism surpasses the above mentioned theme in order to use it as the basis for an ideal of life by claiming that it would have the virtue "d'emplir l'âme de toute vérité, tout savoir et philosophie" (Rabelais, Le Cinquiesme livre, chapter XLV). The unrecognized Traité de bon usage de vin presents the project of "savoir-vivre" in which the author explains the value and the place attribued to wine.

INDEX

Mots-clés : corps, humanisme, littérature, médecine, spirituel

Keywords : body, humanism, literature, medicine, spiritual

\section{AUTEUR}

\section{FRÉDÉRIC-GAEL THEURIAU}

Université de Tours

Fredericgaeltheuriau[at]orange.fr 